\title{
Pseudomonas aeruginosa multirresistente: um problema endêmico no Brasil
}

\author{
Multidrug-resistant Pseudomonas aeruginosa: an endemic problem in Brazil
}

Patrícia R. Neves'; Elsa M. Mamizuka²; Carlos E. Levy³ Nilton Lincopan ${ }^{4}$

\begin{abstract}
unitermos
Pseudomonas aeruginosa

Resistência bacteriana

Carbapenemases

Metilases

Bombas de efluxo

Porinas

\section{resumo}

Relatos mundiais têm documentado a problemática da endemicidade de isolados clínicos de Pseudomonas aeruginosa multirresistente (MDR) aliada a elevados índices de morbidade/mortalidade. No Brasil, surtos de infecção ocasionados por $P$. aeruginosa têm sido relacionados com uma disseminação clonal da espécie. Atualmente, as opções terapêuticas para o tratamento das infecções causadas por esse microrganismo são limitadas, muitas vezes restringindo-se ao uso de carbapenêmicos (p. ex., imipenem [IPM]). Assim, a resistência ao IPM é uma questão de saúde pública, uma vez que esse antibiótico é empregado como último recurso no tratamento de infecções de origem hospitalar, causadas por bactérias Gram-negativas multirresistentes. No Brasil, os principais mecanismos relacionados com fenótipos multirresistentes de P. aeruginosa são produção de metalobetalactamase $(\mathrm{MBL})$ do tipo SPM-1, presença de metilase $16 \mathrm{~S}$ rRNA RmtD, perda de porina OprD e superexpressão de bombas de efluxo, o que pode explicar os altos índices de resistência a carbapenêmicos e aminoglicosídeos. A emergência de cepas com essas características é preocupante, tendo em vista a escassez de terapias efetivas no tratamento de infecções por esse patógeno. Finalmente, com base em relatos nacionais, publicados por diferentes grupos de pesquisa, podemos deduzir que a convergência de múltiplos mecanismos de resistência em P. aeruginosa tem sido um evento favorável para a seleção de diferentes clones endêmicos multirresistentes disseminados no Brasil.
\end{abstract}

Global reports have documented the endemicity of multidrug-resistant (MDR) Pseudomonas aeruginosa associated with high levels of morbidity/mortality. In Brazil, outbreaks of MDR P. aeruginosa have been related to clonal dissemination. Currently, therapeutic options for the treatment of these infections are restricted to carbapenemic antibiotics (i.e., imipenem [IPM]). Thus, carbapenem resistance is a public health issue, since carbapenems are considered the last resort to nosocomial infections caused by MDR Gram-negative bacteria. In Brazil, the main mechanisms associated with MDR P. aeruginosa phenotypes are metallo-betalactamase (MBL) production (SPM-1 enzyme), presence of 16S rRNA methylase RmtD, loss of OprD porin, and overexpression of efflux pumps, which may explain the high level of carbapenem and aminoglycoside resistance. Accordingly, the emergence and dissemination of MDR strains is worrisome. Finally, based on national reports published by different groups of investigators, it is deduced that the convergence of multiple mechanisms of P. aeruginosa resistance has played a major role in the selection of endemic MDR clones widespread in Brazil.

\section{key words}

Pseudomonas aeruginosa

Bacterial resistance

Carbapenemases

Methylases

Efflux pumps

Porins

1. Pós-doutoranda em Microbiologia pelo Instituto de Ciências Biomédicas da Universidade de São Paulo (ICB/USP); doutora em Ciências pela Faculdade de Ciências Farmacêuticas (FCF) da USP. 2. Doutora em Microbiologia e Imunologia; professora da FCF/USP.

3. Doutor em Clínica Médica; professor da Faculdade de Ciências Médicas da Universidade Estadual de Campinas (FCM/UNICAMP).

4. Doutor em Ciências Farmacêuticas; professor do ICB/USP.

Auxilio financeiro: Fundação de Amparo à Pesquisa do Estado de São Paulo (FAPESP). 


\section{Introdução}

Pseudomonas aeruginosa é um dos principais agentes de infecção nosocomial em hospitais brasileiros, onde diversos estudos têm associado sua presença a uma disseminação clonal da espécie ${ }^{(10,20,25,36,63,81,92)}$.

A importância clínica da infecção por $P$. aeruginosa caracteriza-se pela expressão de múltipla resistência a antibacterianos associada a uma difícil erradicação da doença, consequentemente com elevados índices de morbidade e mortalidade $^{(31,53,63) \text {. }}$

Esse microrganismo pode apresentar resistência natural ou adquirida a grande número de antibióticos utilizados na prática clínica ${ }^{(53,114)}$.

O intercâmbio de material genético, que ocorre de forma natural intra ou interespécies entre os bacilos Gram-negativos, é apontado como um dos responsáveis pela aquisição de determinantes de resistência. Assim, a capacidade que $P$. aeruginosa possui de tornar-se resistente durante o tratamento ao antibiótico é inerente à espécie e muitas vezes, inevitável(49).

Nas unidades de terapia intensiva (UTIs) de hospitais brasileiros, a resistência a antibióticos é muito preocupante. Estudos multicêntricos, sejam do SENTRY ou do MISTYC, têm mostrado que a resistência ao imipenem (IPM) é o principal problema encontrado e os índices de resistência em nosso país têm aumentado drasticamente nos últimos anos (Tabela 1).

Estudos epidemiológicos nacionais realizados pelo SENTRY, direcionados a pacientes hospitalizados, avaliaram 3.728 isolados, entre bactérias Gram-positivas e Gramnegativas, obtidos de 12 centros hospitalares de quatro estados, e $P$. aeruginosa foi responsável por $496(13,3 \%)$ casos e o terceiro patógeno mais frequente, com 30,2\% de resistência ao IPM $^{(94)}$. Já o MYSTIC, específico para estudos epidemiológicos em UTIs, avaliou 1.550 amostras de bactérias Gram-negativas, provenientes de 20 centros hospitalares, e $P$. aeruginosa estava envolvida em 30,3\% (470 isolados) das infecções de corrente sanguínea/cateter, trato respiratório, trato urinário, pele/tecidos moles, e com $36,6 \%$ de resistência ao IPM ${ }^{(32)}$. Outros estudos, realizados no Sul e Centro-Oeste do país, relataram percentuais de resistência de $58,9 \%$ até $82,7 \%$, respectivamente ${ }^{(28,121)}$. Esse aumento no perfil de resistência aos antibióticos é uma tendência globalizada, gerando maior impacto na América Latina (Tabela 1).

\section{A importância do IPM na terapia e as consequências da resistência nas infecções por $P$. aeruginosa}

Os antibióticos carbapenêmicos (IPM e meropenem [MEM]) são betalactâmicos de amplo espectro, derivados da tienamicina, com atividade bactericida no tratamento de infecções provocadas por isolados multirresistentes de $P$. aeruginosa. Possuem considerável estabilidade diante da maioria das betalactamases, incluindo as de amplo espectro (ESBL); por essa razão, os carbapenêmicos são considerados fármacos de reserva, frequentemente empregados como último recurso no tratamento de infecções hospitalares causadas por bactérias Gram-negativas resistentes aos demais betalactâmicos ou a outros antibacterianos ${ }^{(84,91)}$.

Assim, como a frequência de $P$. aeruginosa resistente aos carbapenêmicos tem aumentado significativamente e como a indústria farmacêutica não tem lançado uma alternativa terapêutica com um espectro de atividade similar ou superior ao IPM, o atual prognóstico das infecções por bactérias multirresistentes muitas vezes é desfavorável. Nesses casos, o panorama atual da antibioticoterapia restringe-se a alternativas terapêuticas com fármacos considerados inadequados devido à sua alta toxicidade, como, por exemplo, as

Tabela 1 Índice de resistência em Pseudomonas aeruginosa isolada no Brasil

Antibióticos Resistência (\%)

América Latina

SENTRY(3) SENTRY(93)

Imipenem

Meropenem

Amicacina

Ceftazidima

37,8

35,6

34,6

43,7

Ciprofloxacino
50,1
MYSTICY(32)

36,6

25,6

30,5

34,3

41,3

48,3
Brasil

Região

Centro-Oeste ${ }^{(121)}$

58,3

50,0

57,6

48,7

59,5
Região

Sudeste ${ }^{(81)}$

38,3

30,4

35,6

36,5

43,0
Região

Sul(28)
82,7 $-$

78,7

90,7

93,7 
polimixinas (polimixina B e colistina), as quais, muitas vezes, não se encontram disponíveis comercialmente no Brasil(24,41,78).

\section{Principais mecanismos de resistência ao imipenem em $P$. aeruginosa}

A perda de sensibilidade ao IPM pode ser decorrente da: a) perda de porinas; b) presença de proteínas de ligação às penicilinas (PBP) com baixa afinidade por carbapenêmi$\cos ;$ c) superexpressão de bombas de efluxo; d) hidrólise enzimática.

Entre os mecanismos responsáveis pela resistência ao IPM, a impermeabilidade da membrana, devido à perda de porinas ou à superexpressão das bombas de efluxo, confere uma resistência adicional a várias classes de antibióticos como resultado de um efeito cascata gerado por múltiplos mecanismos de resistência inter-relacionados ${ }^{(51,53,71,72)}$.

\section{P. aeruginosa produtora de metalobetalactamase}

Desde 2002, seguindo uma tendência mundial, isolados produtores de metalobetalactamase (MBL) começaram a ser descritos no Brasil, sendo responsáveis pelos elevados níveis de resistência aos carbapenêmicos ${ }^{(26,48,93)}$. À exceção do aztreonam, essas enzimas degradam todos os betalactâmicos, incluindo as associações com inibidores comerciais de betalactamases, como tazobactam, clavulanato e sulbactam. O grupo MBL é representado por enzimas tipo imipenem (IMP) ${ }^{(109)}$, Verona integronencoded metallo-beta-lactamase (VIM) (38), German imipenemase $(\mathrm{GIM})^{(8)}$, Seoul imipenemase $(\mathrm{SIM})^{(40)}$, São Paulo metallo-betalactamase (SPM-1) $)^{(102)}$, Australian imipenemase (AIM) ${ }^{(118)}$, Kyorin health science metallo-beta-lactamase (KHM-1) ${ }^{(97)}$, New Delhi metallo-beta-lactamase (NDM-1) ${ }^{(119)}$, Dutch imipenemase (DIM-1) $^{(85)}$ e Tripoli MBL (TMB-1) $)^{(17)}$.

Em isolados de $P$. aeruginosa produtores de MBL no Brasil, tem sido relatada a presença de IMP e VIM, porém o principal problema se concentra em isolados produtores de enzimas SPM-1, que parecem ser endêmicos e responsáveis por altos índices de mortalidade ${ }^{(21,26,47,64,93,104,122)}$. A identificação das MBLs em $P$. aeruginosa é uma urgência clínica e epidemiológica. Laboratorialmente, a pesquisa de $M B L$ pode ser realizada similarmente à triagem de ESBL nas enterobactérias, porém são utilizados inibidores derivados de ácidos tiólicos ou agentes quelantes, como ácido etilenodiaminotetracético (EDTA), ácido 2-mercaptoacético (MAA) e 2-mercaptopropiônico (MPA) ${ }^{(5)}$. Além do método de aproximação de disco para determinação de $\mathrm{MBL}$, existem fitas comerciais denominadas Etest (AB Biodisk,
Suécia). Redução $\geq 3$ diluições na CIM do imipenem em presença de EDTA é um indicativo da produção de MBL, como demonstrado na Figura $\mathbf{1}^{(58,71,107)}$.

O método de dupla difusão do disco para detecção de isolados produtores de MBL indicou o MPA como agente quelante de maior sensibilidade entre os inibidores de $\mathrm{MBL}$ utilizados (MAA, EDTA, $\mathrm{CuCl}_{2}$ e $\mathrm{FeCl}_{2}$ ), tendo ceftazidima (CAZ) como substrato ${ }^{(5)}$. Posteriormente, foi relatado que o EDTA, em presença do substrato IPM, apresentou maior sensibilidade para detecção de isolados produtores de MBL por meio do teste de sinergismo com disco de $\operatorname{EDTA}^{(39,77)}$. A eficiência do EDTA em baixas concentrações foi relatada para indicar a presença de MBL, aumentando sua eficácia na presença dos substratos CAZ e MEM em isolados produtores de MBL dos tipos VIM e IMP(59). Outros pesquisadores indicaram, em um estudo com número reduzido de amostras avaliadas, o MPA como melhor inibidor ${ }^{(82)}$. Há um único relato avaliando um método de triagem para a detecção de $\mathrm{MBL}$ feito no $\mathrm{Brasil}^{(4)}$. O método utilizado nesse estudo foi o do disco combinado, em que os inibidores foram adicionados diretamente sobre o disco contendo o antibiótico (CAZ e/ou IPM), observando-se mudanças em comparação com o halo de inibição para os discos antibióticos sem adição do inibidor. Um recente estudo, realizado com diversos isolados de $P$. aeruginosa imipenem resistentes clonalmente diferentes, revelou que para a identificação fenotípica de MBL tipo SPM-1, utilizando dupla difusão do disco, a melhor combinação resultou ser MAA-CAZ, ressaltando que o uso de mais de um substrato na presença de mais de um inibidor (EDTA, MPA, IPM) inclui a detecção de MBL do tipo $\operatorname{VIM}^{(71)}$, confirmando dados de estudos com enfoque estrutural e bioquímico da enzima, que têm mostrado que SPM-1 possui comportamento diferente de outras MBLs, mostrando propriedades hidrolíticas totalmente diferentes ${ }^{(70,108)}$. Essa informação é importante, uma vez que SPM-1 é a principal MBL produzida por isolados brasileiros de $P$. aeruginosa, enzima até o momento restrita ao Brasil.

\section{Porinas}

As bactérias Gram-negativas possuem, em sua composição, uma membrana externa que fisiologicamente se caracteriza por ser a primeira linha de defesa contra compostos tóxicos.

O ingresso de substâncias na bactéria é controlado pelas porinas de membrana externa (OMP), que são proteínas capazes de formar canais constituídos de água em seu 


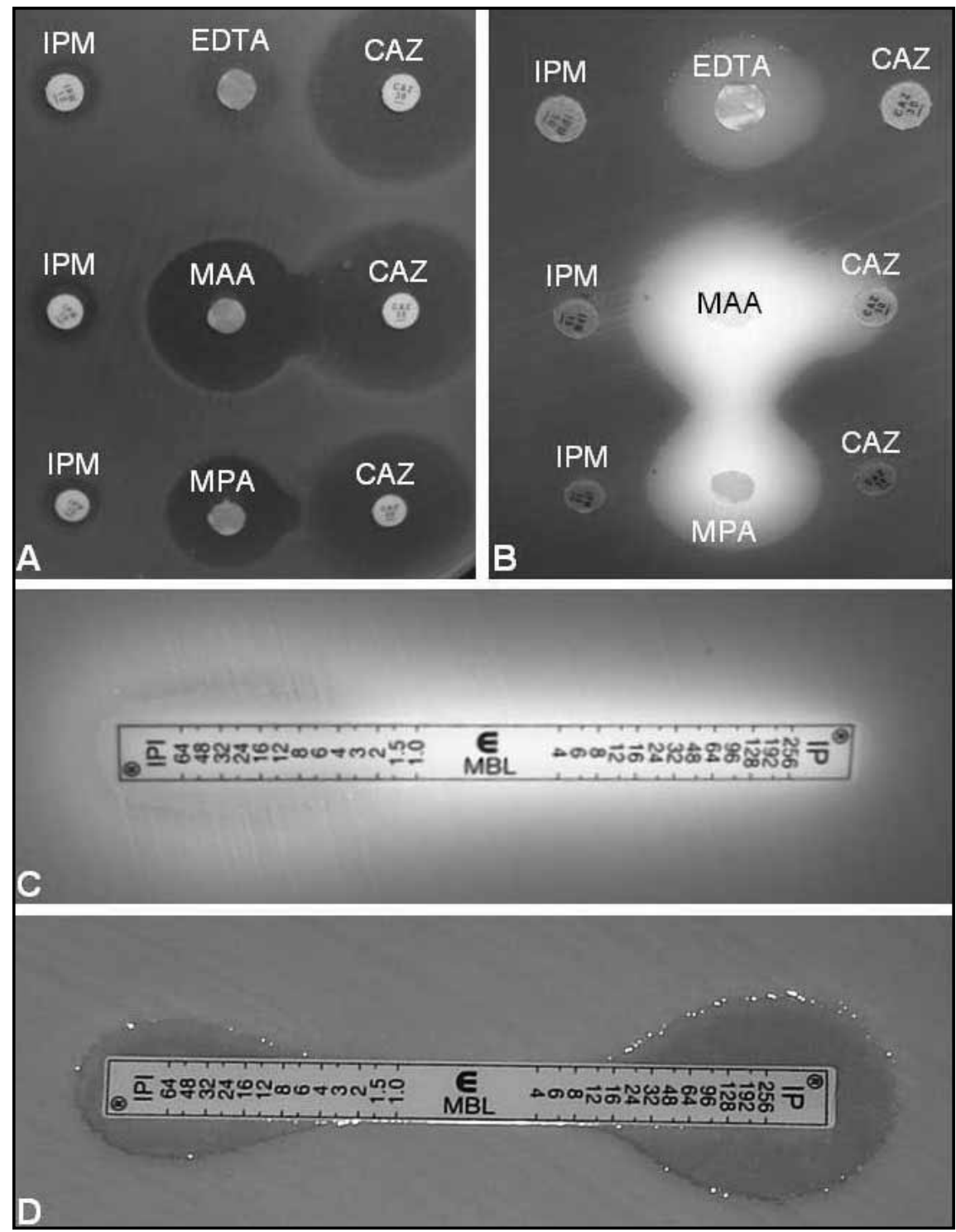

Figura 1 - Método de dupla difusão do disco ( $A$ e B) e método Etest MBL (C e D)

(A) Positividade para MAA-CAZ e MPA-CAZ (formação de zona de inibição de crescimento bacteriano); (B) positividade para EDTA-CAZ, MAA-CAZ e MPA-CAZ; (C) Etest positivo em isolado SPM-1 (decréscimo da CIM de > $256 \mu \mathrm{g} / \mathrm{ml}$ para $4 \mu \mathrm{g} / \mathrm{ml}$ na presença de EDTA); (D) Etest positivo em isolado VIM (decréscimo da CIM de $16 \mu \mathrm{g} / \mathrm{ml}$ para $4 \mu \mathrm{g} / \mathrm{ml}$ na presença de EDTA). Os isolados testados foram obtidos durante um estudo multicêntrico realizado em São Paulo, no período entre 2004 e 2007(71).

MBL: metalobetalactamase; IPM: imipenem; MAA: ácido 2-mercaptoacético; CAZ: ceftazidima; MPA: ácido 2-mercaptopropiônico; EDTA: ácido etilenodiaminotetracético; SPM-1: São Paulo metallo-beta-lactamase; CIM: concentração inibitória mínima; VIM: Verona integron-encoded metallo-beta-lactamase.

interior, o que permite a difusão passiva de solutos hidrofílicos através da membrana externa ${ }^{(74)}$.

As OMP das bactérias Gram-negativas têm sido classificadas e caracterizadas segundo sua atividade, estrutura funcional, regulação e expressão(79).

Em P. aeruginosa, diferentes porinas podem ser encontradas em sua membrana externa, cada qual com sua função, como OprC, OprD, OprE e OprF. Entre elas, a OprF é a mais abundante e, provavelmente, a mais utilizada para difusão da maioria dos betalactâmicos no interior da bactéria ${ }^{(53)}$. OprC e OprE são canais inespecíficos, utilizados por alguns antimicrobianos ${ }^{(105)}$. A presença de OprD, também denominada porina $\mathrm{D} 2$, tem sido amplamente investigada, uma vez que a ausência ou mesmo a expressão reduzida do gene oprD, codificador dessa porina, tem contribuído para a resistência aos carbapenêmicos, especialmente IPM (Tabela 2) $23,29,54,75,115-117,120)$.

Devido à falta de consenso sobre a caracterização dessa proteína com determinado peso molecular, podemos encontrar relatos da ausência de proteínas OprD de 42 até 
Interpretação fenotípica de mecanismos de impermeabilidade associados à produção de MBL em Pseudomonas

Tabela 2 aeruginosa*

\begin{tabular}{cccc} 
IPM & IPM/EDTA & MBL & Porina OprD \\
$\mathrm{S}$ & $\mathrm{S}$ & - & + \\
$\mathrm{I} / \mathrm{R}$ & $\mathrm{I} / \mathrm{R}$ & - & - \\
$\mathrm{R}$ & $\mathrm{R}$ & + & - \\
$\mathrm{R}$ & $\mathrm{S}$ & + & + \\
\hline
\end{tabular}

MBL: metalobetalactamase; IPM: imipenem; EDTA: ácido etilenodiaminotetracético; S: sensível; I: intermediário; R: resistente; (-): negativo/ausente; $(+)$ : positivo/presente.

${ }^{*}$ Adaptado de Livermore (2003)(53).

$54 \mathrm{kDa}^{(23,52,55,69,73,75,103,120)}$ com fenótipos IPM resistentes. De fato, existem relatos que indicam a existência de homólogos da proteína OprD, o que pode gerar produtos de expressão de tamanhos diferenciados ${ }^{(76,100,101)}$. Além disso, a ausência dessa proteína tem sido atribuída à presença de sequências de inserção no gene estrutural $\left.\right|^{(18,71,110)}$.

Fisiologicamente, a principal função da porina OprD é permitir a captação passiva dos aminoácidos básicos através da membrana externa. Sabe-se que ela é capaz de permitir a entrada de carbapenêmicos, ainda que não de outros betalactâmicos ${ }^{(120)}$. A afinidade e a capacidade de difusão de IPM por meio dessa porina são quase 70 vezes mais elevadas que as observadas para $\mathrm{MEM}^{(75)}$. Isolados que apresentam mutações na porina OprD são selecionados durante o tratamento com IPM. Esses isolados demonstram diminuição da afinidade e do transporte desse antibiótico por essa proteína, comprovando, assim, que a resistência ao IPM está intimamente ligada à falta de expressão ou perda da OprD, mostrando aumento da CIM para IPM, com valores que atingem um limiar característico de resistência intermediária ${ }^{(23,29,54,75,115-117)}$.

A resistência ao MEM está ligada à mutação do gene que codifica a porina OprD e à ativação de bombas de efluxo que utilizem o MEM como substrato. A mutação do gene oprD ocorre em isolados resistentes ao IPM com sensibilidade reduzida ou preservada ao MEM, sem afetar outros betalactâmicos, a menos que outros mecanismos de resistência estejam presentes ${ }^{(33,54,76)}$.

Alguns autores têm relatado a presença de resistência cruzada a betalactâmicos, aminoglicosídeos e quinolonas após o uso de ciprofloxacino. Essa resistência está associada a alterações nas proteínas da membrana externa, incluindo aumento da expressão de proteínas de $54 \mathrm{kDa}^{(55,69,90)}$. Outros estudos descreveram um isolado resistente a quinolonas e IPM, que foi obtido da urina de um paciente tratado com norfloxacino. Foram atribuídos a esse isolado mecanismos de resistência relacionados com alterações na DNA girase (gene $n f x C$ ), perda da porina OprD (46 kDa) e aumento de proteína de $50 \mathrm{kDa}^{(22,62,88)}$.

\section{Superexpressão de bombas de efluxo}

A maioria dos mecanismos de proteção bacteriana contra o ingresso indesejado de moléculas prejudiciais à célula é governada por um mecanismo de transporte ativo para o exterior celular, por meio de um sistema denominado bomba de efluxo ${ }^{(96)}$. Esses sistemas de expulsão são os responsáveis pela "impermeabilidade" à maioria dos antibióticos e desinfetantes em grande variedade de espécies bacterianas ${ }^{(6,43,45,50,83)}$. A principal função das bombas de efluxo presentes em $P$. aeruginosa é exportar substâncias tóxicas ou metabólitos secundários, assim como a excreção de moléculas sinalizadoras que governam a comunicação celular ${ }^{(37,80)}$. Os antibióticos estão entre esses compostos tóxicos e a extrusão dos mesmos compromete a eficácia terapêutica. Muitos genes codificadores de bombas de efluxo para múltiplos antimicrobianos são expressos constitutivamente, conferindo resistência intrínseca aos mesmos ${ }^{(57)}$.

Os sistemas de efluxo de $P$. aeruginosa pertencem à família resistance-nodulation-cell division (RND) ${ }^{(56,57,83)}$. Esses sistemas têm como base a abertura de um canal que atravessa as membranas interna e externa da bactéria, permitindo a remoção de moléculas para o exterior celular.

O genoma de $P$. aeruginosa contém genes que codificam sistemas de efluxo da família RND denominados multidrug efflux pump (Mex), dos quais dez já foram caracterizados: MexAB-OprM( ${ }^{(88)}$, MexCD-Opr] ${ }^{(89)}$, MexEF-OprN ${ }^{(35)}$, MexXYOprM(68), MexGHI, OpmD(1), MexVW-OprM(46), MexPQOpmE ${ }^{(66)}$, MexMN-OprM( ${ }^{(66)}$ e TriABC-OpmH ${ }^{(67)}$. Esses sistemas são denominados multidrug-resistance (MDR), pois são capazes de conferir resistência a um amplo espectro de quimioterápicos ${ }^{(37,83)}$.

Entre as bombas de efluxo caracterizadas em $P$. aeruginosa, a expressão de quatro sistemas (MexABOprM, MexCD-Opr], MexEF-OprN e MexXY-OprM) foi relatada entre isolados clínicos e relacionados com a resistência a múltiplos antibióticos (Tabela 3) $)^{(2,50,61)}$.

As bombas de efluxo de bactérias Gram-negativas constituem um sistema tripartite de proteínas de transporte que se localizam ao longo da membrana interna (proteína constituída por uma bomba de transporte ativo, 
Substratos antimicrobianos para diferentes sistemas de efluxo presentes em isolados

Tabela 3 clínicos de Pseudomonas aeruginosa*

\begin{tabular}{|c|c|c|c|c|}
\hline \multirow{2}{*}{ Substrato antibiótico } & \multicolumn{4}{|c|}{ Sistema de efluxo } \\
\hline & MexA-MexB-OprM & MexC-MexD-OprJ & MexE-MexF-OprN & MeXX-MexY-OprM \\
\hline Amicacina & & & & $x$ \\
\hline Aztreonam & $x$ & & & \\
\hline Carbenicilina & $x$ & & & \\
\hline Cefepima & & $x$ & & $x$ \\
\hline Cefotaxima & $x$ & & & $x$ \\
\hline Ceftazidima & $x$ & & & \\
\hline Cefuroxima & $x$ & $x$ & & \\
\hline Ciprofloxacino & $x$ & $x$ & $x$ & $x$ \\
\hline Clavulanato & $x$ & & $x$ & \\
\hline Cloranfenicol & $x$ & $x$ & $x$ & \\
\hline Eritromicina & & $x$ & & $x$ \\
\hline Faropenem & $x$ & & & \\
\hline Gentamicina & & & & $x$ \\
\hline Imipenem & & & $x$ & \\
\hline Levofloxacino & $x$ & $x$ & $x$ & $x$ \\
\hline Meropenem & $x$ & & & \\
\hline Nafcilina & $x$ & $x$ & & \\
\hline Norfloxacino & $x$ & $x$ & $x$ & \\
\hline Piperacilina & $x$ & & & \\
\hline Sulbactam & $x$ & & $x$ & \\
\hline Tetraciclina & $x$ & $x$ & & $x$ \\
\hline Tobramicina & & & & $x$ \\
\hline Trimetoprima & $x$ & & $x$ & \\
\hline Trovafloxacino & & $x$ & & \\
\hline
\end{tabular}

Mex: multidrug efflux pump.

*Adaptado de Aeschlimann (2003)(2).

dependente de energia - MexB, MexD, MexF ou MexY), periplasma (proteína transmembrana que une os outros dois componentes - MexA, MexC, MexE ou MexX) e membrana externa (proteína formadora de canal extrusor, também denominada porina - OprJ, OprM ou OprN) (Figura 2).

A habilidade do sistema de efluxo em reconhecer grande número de compostos é provavelmente devido às suas propriedades físico-químicas, como hidrofobicidade e aromaticidade, e ao caráter ionizável ${ }^{(96)}$. Os antibióticos, em sua maioria, são anfifílicos e possuem características hidrofílicas e hidrofóbicas, sendo facilmente reconhecidos por muitas bombas de efluxo ${ }^{(30)}$, podendo, inclusive, agir como reguladores da expressão de alguns sistemas de efluxo, como no caso do ciprofloxacino(53).
Cada bomba tem afinidade por um substrato antimicrobiano e as fluoroquinolonas são substratos universais para todas as bombas do tipo Mex (Tabela 3).

Entre os substratos preferenciais das bombas de efluxo presentes em isolados clínicos de $P$. aeruginosa, os carbapenêmicos estão presentes nos sistemas MexAB-OprM e MexEF-OprN.

MexAB-OprM foi a primeira bomba de efluxo da família RND caracterizada em $P$. aeruginosa ${ }^{(88)}$. Esse sistema compromete a ação de vários antibacterianos, inclusive MEM, mas não inclui o IPM devido a diferenças em sua estrutura molecular ${ }^{(33,42,44,53,60)}$. A expressão desse sistema é regulada pela inserção da proteína MexR na região entre os genes $\operatorname{mexR}$ e mexA (região promotora), atuando, assim, como repressora (Figura 3). 


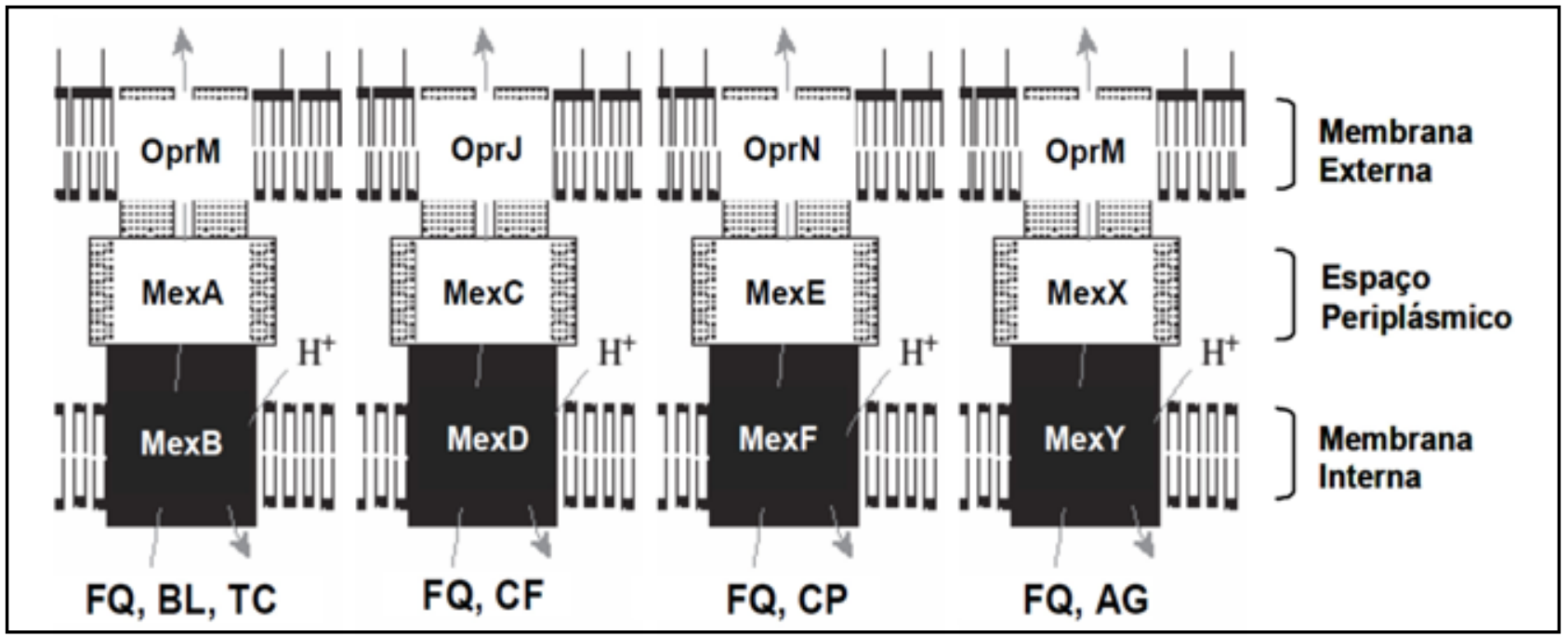

Figura 2 - Modelos estruturais e funcionais das bombas de efluxo de P. aeruginosa, indicando a localização das proteínas constituintes da bomba e ação preferencial a determinados tipos de substratos

FQ: fluoroquinolona; BL: betalactâmico; TC: tetraciclina; CF: cefalosporina; CP: carbapenêmico; AG: aminoglicosídeo. Adaptada de Aeschlimann (2003)(2), Neves (2010)(71) e Poole (2005)(86).

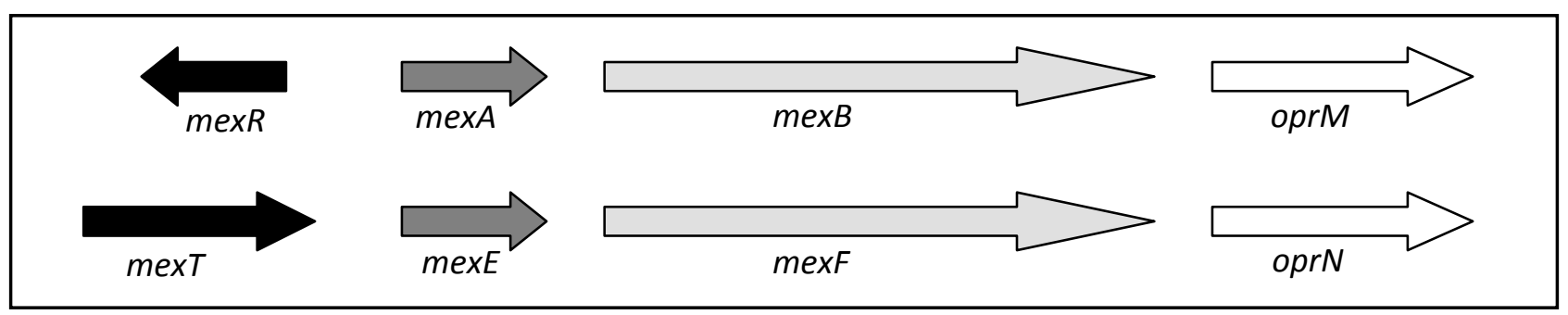

Figura 3 - Contexto genético de genes codificadores de proteínas constituintes dos sistemas de efluxo MexAB-OprM e MexEF-OprN em P. aeruginosa Cada operon contém os genes que codificam os componentes dos sistemas de efluxo: regulador transcricional (preto) e proteínas de membrana interna (cinza claro), periplasmática (cinza escuro) e de membrana externa (branco). Ambos os sistemas são responsáveis por fenótipos carbapenem-resistentes na espécie estudada. Adaptada de Lister et al. (2009)(50).

MexEF-OprN está relacionado com resistência ao IPM, pois sua expressão é dependente da presença da proteína MexT (codificada pelo gene mexT), que atua como ativadora pós-transcricional ${ }^{(34)}$. Além de ativar a expressão do sistema, acredita-se que MexT possa atuar como repressora da expressão da proteína OprD, pois está envolvida na mutação que origina a perda dessa porina ${ }^{(35)}$. Além disso, esse sistema pode diminuir a expressão das bombas MexAB-OprM e MexXY-OprM, pois parece possuir atividade corregulatória sobre elas ${ }^{(60,87,98,99)}$.

As bombas de efluxo são codificadas por elementos genéticos localizados em cromossomos e em algumas espécies elas têm sido detectadas em plasmídeos, o que pode facilitar a propagação dos genes de efluxo, contribuindo para a resistência intrínseca e adquirida, permitindo à bactéria sobreviver em ambientes hostis, como, por exemplo, na presença de antibióticos e desinfetantes ${ }^{(7,86)}$.
A organização genética das bombas de efluxo da família RND em $P$. aeruginosa é governada por operons. Cada operon contém os genes que codificam os componentes dos sistemas de efluxo (proteínas de membrana interna, periplasmática e de membrana externa), conforme demonstrado na Figura 3.

O aumento da expressão das bombas de efluxo, ou superexpressão, pode ser mediado por mutações (em um gene regulador proximal $[\operatorname{mexR}, \operatorname{mex} T]$ ou na região promotora do gene transportador [mexB, mexF]) ou por elementos de inserção localizados na região anterior ao gene transportador ${ }^{(83)}$.

A presença de sequências de inserção de um componente estrutural do sistema de efluxo na região anterior aos genes codificadores, ou inseridas no gene repressor, tem sido descrita em isolados que superexpressam bombas de efluxo que conferem multirresistência aos antibióticos $(\mathrm{MDR})^{(30,83)}$. 
Os mecanismos envolvidos na superexpressão das bombas de efluxo têm sido pouco estudados, pois são difíceis de serem inferidos, a priori, pelos resultados dos testes de sensibilidade qualitativos, semiquantitativos ou quantitativos (disco-difusão, sistemas automatizados e determinação da CIM).

Uma estratégia para a pesquisa laboratorial de bombas de efluxo tem sido baseada no uso de inibidores de sistemas de efluxo combinados com substratos antimicrobianos $^{(11,57)}$.

Os inibidores de bombas de efluxo geralmente são alcaloides de plantas. Entre eles, podemos citar o cianeto de carbonila m-clorofenil-hidrazona (CCCP), o dinitrofenol (DNP), a reserpina e o fenil-arginina-betanaftilamida (PABN). Esses inibidores são amplamente utilizados como ferramenta na detecção de bombas de efluxo ${ }^{(11,37,57)}$.

Outra metodologia confiável e sensível para investigar os sistemas de efluxo é a reação em cadeia da polimerase (PCR) em tempo real, que permite identificar o nível de expressão dos genes de interesse e tem sido empregada na detecção da expressão de sistemas de efluxo de $P$. aeruginosa, incluindo investigações realizadas por grupos de pesquisa no Brasil $(19,65,71,111)$.

\section{Produção de metilases 165 rRNA e resistência aos aminoglicosídeos}

Diferentemente das betalactamases, as enzimas que conferem resistência aos aminoglicosídeos agem modificando quimicamente a estrutura do antibiótico, antes que este se ligue a seu alvo (subunidades do ribossomo).

A modificação enzimática pode afetar tanto grupos aminas como hidroxilas, mediante processos de O-fosforilação ou O-adenilação por fosfotransferases (APH) e nucleotidiltransferases (ANT) dependentes de ATP ou por meio de um processo de $\mathrm{N}$-acetilação por acetiltransferases (AAC) dependentes de acetil coenzima A (acetil-coA). Desse grupo de enzimas, aquelas citadas como $\operatorname{AAC}\left(6^{\prime}\right)$, ANT( $\left.2^{\prime \prime}\right)$ e $\mathrm{APH}\left(3^{\prime}\right)$ têm sido as mais frequentemente descritas em isolados de $P$. aeruginosa resistentes a antibióticos, como gentamicina, tobramicina, amicacina e neomicina ${ }^{(9)}$.

Outro grupo de enzimas que conferem resistência mediada pela metilação sítio-específica do RNA ribossômico $16 S$ é conhecido como metilases $16 \mathrm{~S}$ rRNA ${ }^{(112,113)}$. Inicialmente, acreditava-se que esse mecanismo de resistência não existia em espécies clínicas relevantes, porém, foram relatados isolados de $P$. aeruginosa que produziam metilases $16 \mathrm{~S} r \mathrm{rNA}^{(12,27,112)}$. Essas enzimas se mostraram capazes de conferir nível elevado de resistência a aminoglicosídeos utilizados clinicamente, como amicacina, tobramicina e gentamicina ${ }^{(12-14,16,47)}$.

Existem relatos de cinco tipos de metilases $16 \mathrm{~S}$ rRNA até o momento: aminoglycoside resistance methyltransferase $(\operatorname{ArmA})^{(27)}$, ribosomal methyltransferase A $(\operatorname{RmtA})^{(113)}$, $\mathrm{RmtB}^{(15)}, \mathrm{RmtC}^{(106)}$ e, mais recentemente, $\mathrm{RmtD}^{(12)}$. Essa metilase foi encontrada em uma amostra clínica de $P$. aeruginosa isolada no Brasil, produtora de $\mathrm{MBL}$ do tipo SPM-1. Fenotipicamente, esse isolado apresentou resistência a todos os betalactâmicos, incluindo os carbapenêmicos, e aminoglicosídeos, tornando inviável o uso de um esquema terapêutico sinérgico com base nessa associação.

Com relação a esquemas terapêuticos usando aminoglicosídeos, a monoterapia com esses antibióticos, como amicacina e gentamicina, raramente é utilizada. Alguns protocolos recomendam para o tratamento de infecções graves por $P$. aeruginosa, principalmente no caso de bacteremia, a utilização combinada de betalactâmicos e aminoglicosídeos, a fim de minimizar os riscos de falha na terapêutica devido à resistência ${ }^{(95)}$. Porém, no Brasil, para ambos os antibióticos, os índices de resistência têm aumentado drasticamente nos últimos anos (Tabela 1), existindo relatos de isolados capazes de coproduzir enzimas ESBL ou MBL, juntamente com metilases, contribuindo para o aparecimento de isolados multirresistentes $^{(12,14,16)}$.

\section{Conclusão}

Os principais mecanismos relacionados com fenótipos multirresistentes de $P$. aeruginosa em hospitais brasileiros são produção de MBL do tipo SPM-1 e de metilase 16S rRNA RmtD, perda de porina OprD e superexpressão de bombas de efluxo, o que pode explicar os altos índices de resistência a carbapenêmicos e aminoglicosídeos. A emergência de isolados com essas características é preocupante e ocasiona grande impacto clínico, tendo em vista a escassez de terapias efetivas no tratamento de infecções por esse patógeno. Culturas de vigilância e a precoce deteç̧ão de isolados com fenótipo multirresistente poderão contribuir para um controle epidemiológico efetivo, evitando a instauração de surtos, sendo que a convergência de múltiplos mecanismos de resistência em $P$. aeruginosa parece ser um evento favorável para a seleção de diferentes clones endêmicos multirresistentes disseminados no Brasil. 


\section{Referências}

1. AEDEKERK, S. et al. Characterization of a new efflux pump, MexGHI-OpmD, from Pseudomonas aeruginosa that confers resistance to vanadium. Microbiology, v. 148, n. 8, p. 2371-81, 2002.

2. AESCHLIMANN, J. R. The role of multidrug efflux pumps in the antibiotic resistance of Pseudomonas aeruginosa and other Gram-negative bacteria. Pharmacotherapy, v. 23, n. 7, p. 916-24, 2003.

3. ANDRADE, S. S. et al. Increasing prevalence of antimicrobial resistance among Pseudomonas aeruginosa isolates in Latin American medical centres: 5 year report of the SENTRY Antimicrobial Surveillance Program (1997-2001). J Antimicrob Chemother, v. 52, n. 1, p. 140-1, 2003.

4. ANDRADE, S. S. et al. Influence of disk preparation on detection of metallo-\{beta\}-lactamase-producing isolates by the combined disk assay. J Clin Microbiol, v. 45, n. 6, p. 2058-60, 2007.

5. ARAKAWA, Y. et al. Convenient test for screening metalloß-lactamase-producing Gram-negative bacteria by using thiol compounds. J Clin Microbiol, v. 38, n. 1, p. 40-3, 2000

6. BLAIR, J. et al. Structure, function and inhibition of RND efflux pumps in Gram-negative bacteria: an update. Current Opinion in Microbiology, v. 12, n. 5, p. 512-9, 2009.

7. BUTAYE, P. et al. Mobile genes coding for efflux-mediated antimicrobial resistance in Gram-positive and Gramnegative bacteria. Int J Antimicrob Agents, v. 22, n. 3, p. 205-10, 2003.

8. CASTANHEIRA, M. et al. Molecular characterization of a $\beta$-lactamase gene, blaGIM-1, encoding a new subclass of metallo-beta-lactamase. Antimicrobial Agents and Chemotherapy, v. 48, n. 12, p. 4654-61, 2004.

9. DAVIES, J.; WRIGHT, G. D. Bacterial resistance to aminoglycoside antibiotics. Trends Microbiol, v. 5, n. 6, p. 234-40, 1997.

10. DE FREITAS, A. L.; BARTH, A. L. Antibiotic resistance and molecular typing of Pseudomonas aeruginosa: focus on imipenem. Braz J Infect Dis, v. 6, n. 1, p. 1-7, 2002.

11. DENNY, B. J. et al. Antimicrobial activity of a series of 1-alkyl-2-(4-pyridyl)pyridinium bromides against Grampositive and Gram-negative bacteria. Med Princ Pract, v. 14, n. 6 , p. $377-81,2005$.

12. DOI, Y. et al. Coproduction of Novel $16 \mathrm{~S}$ rRNA methylase RmtD and metallo- $\beta$-lactamase SPM- 1 in a panresistant Pseudomonas aeruginosa isolate from Brazil. Antimicrob Agents Chemother, v. 51, n. 3, p. 852-6, 2007a.

13. DOI, Y. et al. Genetic environment of $16 \mathrm{~S}$ rRNA methylase gene rmtD. Antimicrob Agents Chemother, v. 52, n. 6, p. 2270-2. 2008.

14. DOI, Y. et al. High prevalence of metallo-beta-lactamase and $16 \mathrm{~s}$ ribosomal RNA methylase co-production among imipenem-resistant Pseudomonas aeruginosa in Brazil. Antimicrob Agents Chemother, v. 51, n. 9, p. 3388-90, 2007b.

15. DOI, Y. et al. Plasmid-mediated $16 \mathrm{~S}$ rRNA methylase in Serratia marcescens conferring high-level resistance to aminoglycosides. Antimicrob Agents Chemother, v. 48, n. 2, p. 491-6, 2004
16. DOI, Y.; ARAKAWA,Y. 16S ribosomal RNA methylation: emerging resistance mechanism against aminoglycosides. Clin Infect Dis, v. 45, n. 1, p. 88-94, 2007.

17. EL SALABI, A. et al. Novel subclase of a group B1 metallobeta-lactamase, $T M B-1$, in clinical and non-clinical Gram-negative bacteria from Libya. $49^{\text {th }}$ Interscience Conference in Antimicrobial Agentes and Chemotherapy (ICAAC). San Francisco, CA, USA, 2009. n. C1-1365.

18. EVANS, J. C.; SEGAL, H. A novel insertion sequence, ISPa25, in oprD of Pseudomonas aeruginosa associated with carbapenem resistance. Antimicrob Agents Chemother, v. 51, n. 10, p. 3776-7, 2007.

19. FEHLBERG, L. C. C. Estudo comparativo dos mecanismos de resistência aos beta-lactâmicos em amostras clínicas de Pseudomonas aeruginosa isoladas de infecção de corrente sanguínea no Brasil e nos Estados Unidos da América. 2010. Dissertação (Mestrado) - Escola Paulista de Medicina, Universidade Federal de São Paulo.

20. FIGUEIREDO-MENDES, C. M. et al. Pseudomonas aeruginosa clonal dissemination in Brazilian intensive care units. Enferm Infecc Microbiol Clin, v. 23, n. 7, p. 402-5, 2005.

21. FILHO, L. S. et al. Determinação da produção de metalo$B$-lactamases em amostras de $P$. aeruginosa isoladas em João Pessoa, Paraíba. J Bras Patol Med Lab, v. 38, n. 4, p. 291-6, 2002

22. FUKUDA, $\mathrm{H}$. et al. nfxC-type quinolone resistance in a clinical isolate of Pseudomonas aeruginosa. Antimicrobial Agents and Chemotherapy, v. 39, n. 3, p. 790-2, 1995.

23. FUNG-TOMC, J. C. et al. Activity of carbapenem BMS181139 against Pseudomonas aeruginosa is not dependent on porin protein D2. Antimicrob Agents Chemother, v. 39, n. 2, p. 386-93, 1995.

24. FURTADO, G. H. et al. Intravenous polymyxin B for the treatment of nosocomial pneumonia caused by multidrug-resistant Pseudomonas aeruginosa. Int J Antimicrob Agents, v. 30, n. 4, p. 315-9, 2007.

25. GALES, A. C. et al. Carbapenem-resistant Pseudomonas aeruginosa outbreak in an intensive care unit of a teaching hospital. Braz J Infect Dis, v. 8, n. 4, p. 267-71, 2004.

26. GALES, A. C. etal. Dissemination in distinct Brazilian regions of an epidemic carbapenem-resistant Pseudomonas aeruginosa producing SPM metallo-beta-lactamase. J Antimicrob Chemother, v. 52, n. 4, p. 699-702, 2003.

27. GALIMAND, M. et al. Plasmid-mediated high-level resistance to aminoglycosides in Enterobacteriaceae due to $16 S$ rRNA methylation. Antimicrob Agents Chemother, v. 47, n. 8, p. 2565-71, 2005.

28. GONÇALVES, D. C. et al. Detection of metallo-betalactamase in Pseudomonas aeruginosa isolated from hospitalized patients in Goiânia, state of Goiás. Rev Soc Bras Med, v. 42, n. 4, p. 411-4, 2009

29. HUANG, H.; HANCOCK, R. E. The role of specific surface loop regions in determining the function of the imipenem-specific pore protein OprD of Pseudomonas aeruginosa. J Bacteriol, v. 178, n. 11, p. 3085-90, 1996. 
30. KAATZ, G. W. Inhibition of bacterial efflux pumps: a new strategy to combat increasing antimicrobial agent resistance. Expert Opin Emerg Drugs, v. 7, n. 2, p. 223-33, 2002.

31. KHAN, T. Z. et al. Early pulmonary inflammation in infants with cystic fibrosis. Am J Respir Crit Care Med, v. 151, n. 4, p. 1075-82, 1995.

32. KIFFER, C. et al. Antimicrobial susceptibility of Gramnegative bacteria in Brazilian hospitals: the MYSTIC Program Brazil 2003. Braz J Infect Dis, v. 9, n. 3, p. 216-24, 2005.

33. KÖHLER, T. et al. Carbapenem activities against Pseudomonas aeruginosa: respective contributions of OprD and efflux systems. Antimicrob Agents Chemother, v. 43, n. 2, p. 424-7, 1999a.

34. KÖHLER, T. et al. Characterization of MexT, the regulator of the MexE-MexF-OprN multidrug efflux system of Pseudomonas aeruginosa. J Bacteriol, v. 181, n. 20, p. 6300-5, 1999b.

35. KÖHLER, T. et al. Differential selection of multidrug efflux system by quinolones in Pseudomonas aeruginosa. Antimicrob Agents Chemother, v. 41, n. 11, p. 2540-3, 1997.

36. KOKIS, V. M. et al. Identification of an imipenem-resistant Pseudomonas aeruginosa clone among patients in a hospital in Rio de Janeiro. J Hosp Infect, v. 60, n. 1 , p. 19-26, 2005.

37. KRIENGKAUYKIAT, J. et al. Use of an efflux pump inhibitor to determine the prevalence of efflux pump-mediated fluoroquinolone resistance and multidrug resistance in Pseudomonas aeruginosa. Antimicrob Agents Chemother, v. 49, n. 2, p. 565-70, 2005.

38. LAURETTI, L. et al. Cloning and characterization of bla ${ }_{\text {VIM }}$ a new integron-borne metallo-beta-lactamase gene from a Pseudomonas aeruginosa clinical isolate. Antimicrob Agents Chemother, v. 43, n. 7, p. 1584-90, 1999.

39. LEE, K. et al. Evaluation of the Hodge test and the imipenem-EDTA double-disk synergy test for differentiating metallo-beta-lactamase-producing isolates of Pseudomonas spp. and Acinetobacterspp. J Clin Microbiol, v. 41, n. 10, p. 4623-9, 2003.

40. LEE, K. et al. Novel acquired metallo-beta-lactamase gene, blaSIM-1, in a class 1 integron from Acinetobacter baumannii clinical isolates from Korea. Antimicrob Agents Chemother, v. 49, n. 11, p. 4485-91, 2005.

41. LEVIN, A. S. et al. Intravenous colistin as therapy for nosocomial infections caused by multidrugresistant Pseudomonas aeruginosa and Acinetobacter baumannii. Clin Infect Dis, v. 28, n. 5, p. 1008-11, 1999.

42. LI, X. et al. Contributions of MexAB-OprM and an EmrE homolog to intrinsic resistance of Pseudomonas aeruginosa to aminoglycosides and dyes. Antimicrob Agents Chemother, v. 47, n. 1, p. 27-33, 2003b.

43. LI, X. et al. Interplay between the MexA-MexB-OprM multidrug efflux system and the outer membrane barrier in the multiple antibiotic resistance of Pseudomonas aeruginosa. J Antimicrob Chemother, v. 45, n. 4, p. 433-6, 2000.

44. LI, X. et al. Role of efflux pump(s) in intrinsic resistance of Pseudomonas aeruginosa: resistance to tetracycline, chloramphenicol, and norfloxacin. Antimicrob Agents Chemother, v. 38, n. 8, p. 1732-41, 1994.

45. LI, X.; NIKAIDO, H. Efflux-mediated drug resistance in bacteria an update. Drugs, v. 69, n. 12, p.1555-623, 2009.

46. LI, Y. et al. A new member of the tripartite multidrug efflux pumps, MexVW-OprM, in Pseudomonas aeruginosa. J Antimicrob Chemother, v. 52, n. 4, p. 572-5, 2003 a.

47. LINCOPAN, N. et al. Balanoposthitis caused by Pseudomonas aeruginosa co-producing metallo-betalactamase and 16S rRNA methylase in children with hematological malignancies. Int J Infect Dis, v. 14, n. 4, p. e344-7, 2010.

48. LINCOPAN, N. et al. First isolation of metallo-betalactamase-producing multiresistant Klebsiella pneumoniae from a patient in Brazil. J Clin Microbiol, v. 43, n. 1, p. 516-9, 2005.

49. LINCOPAN, N.; TRABULSI, L. R. Pseudomonas aeruginosa. In: TRABULSI, L. R.; ALTERTHUM, F. Microbiologia. 5. ed. São Paulo: Atheneu, 2008. p. 369-81.

50. LISTER, P. D. et al. Antibacterial-resistant Pseudomonas aeruginosa: clinical impact and complex regulation of chromosomally encoded resistance mechanisms. Clin Microbiol Rev, v. 22, n. 4, p. 582-610, 2009

51. LIVERMORE, D. M. Bacterial resistance: origins, epidemiology, and impact. Clin Infect Dis, v. 36, Suppl. 1, p. S11-23, 2003.

52. LIVERMORE, D. M. Interplay of impermeability and chromosomal beta-lactamase activity in imipenemresistant Pseudomonas aeruginosa. Antimicrob Agents Chemother, v. 36, n. 9, p. 2046-8, 1992.

53. LIVERMORE, D. M. Multiple mechanisms of antimicrobial resistance in Pseudomonas aeruginosa: our worst nightmare? Clin Infect Dis, v. 34, n. 5, p. 634-40, 2002.

54. LIVERMORE, D. M. Of Pseudomonas, porins, pumps and carbapenems. J Antimicrob Chemother, v. 47, n. 3, p. 247-50, 2001

55. LIVERMORE, D. M.; WOODFORD, N. Carbapenemases: a problem in waiting? Current Opinion in Microbiology, v. 3, n. 5, p. 489-95, 2000.

56. LLANES, C. et al. Clinical strains of Pseudomonas aeruginosa overproducing MexAB-OprM and MexXY efflux pumps simultaneously. Antimicrob Agents Chemother, v. 48, n. 5, p. 1797-802, 2004.

57. LOMOVSKAYA, O. et al. Identification and characterization of inhibitors of multidrug resistance efflux pumps in Pseudomonas aeruginosa: novel agents for combination therapy. Antimicrob Agents Chemother, v. 45, n. 1, p. 105-16, 2001

58. LUZZARO, F. et al. Prevalence and characterization of metallo-beta-lactamases in clinical isolates of Pseudomonas aeruginosa. Diagn Microbiol Infect Dis, v. 48, n. 2, p. 131-5, 2004.

59. MARCHIARO, P. et al. Sensitive EDTA-based microbiological assays for detection of metallo- $\beta$-lactamases in nonfermentative gram-negative bacteria. J Clin Microbiol, v. 43, n. 11, p. 5648-52, 2005.

60. MASEDA, H. et al. Assignment of the substrate-selective subunits of the MexEF-OprN multidrug efflux pump of Pseudomonas aeruginosa. Antimicrob Agents Chemother, v. 44, n. 3, p. 658-64, 2000. 
61. MASUDA, N. et al. Substrate specificities of MexABOprM, MexCD-OprJ, and MexXY-OprM efflux pumps in Pseudomonas aeruginosa. Antimicrob Agents Chemother, v. 44, n. 12, p. 3322-7, 2000.

62. MASUDA, N.; OHYA, S. Cross-resistance to meropenem, cephems, and quinolones in Pseudomonas aeruginosa. Antimicrob Agents Chemother, v. 36, n. 9, p. 1847-51, 1992.

63. MENDES, C. et al. Antimicrobial susceptibility in intensive care units: MYSTIC Program Brazil 2002. Braz J Infect Dis, v. 9, n. 1, p. 44-51. 2005.

64. MENDES, R. E. et al. Metalo- $\beta$-lactamases. J Bras Patol Med Lab, v. 42, n. 2, p. 103-13, 2006.

65. MESAROS, N. et al. A combined phenotypic and genotypic method for the detection of Mex efflux pumps in Pseudomonas aeruginosa. J Antimicrob Chemother, v. 59, n. 3, p. 378-86, 2007.

66. MIMA, T. et al. Gene cloning and properties of the RND-type multidrug efflux pumps MexPQ-OpmE and MexMN-OprM from Pseudomonas aeruginosa. Microbiol Immunol, v. 49, n. 11, p. 999-1002, 2005.

67. MIMA, T. et al. Identification and characterization of TriABC-OpmH, a triclosan efflux pump of Pseudomonas aeruginosa requiring two membrane fusion proteins. J Bacteriol, v. 189, n. 21, p. 7600-9, 2007.

68. MINE, T. et al. Expression in Escherichia coli of a new multidrug efflux pump, MexXY, from Pseudomonas aeruginosa. Antimicrob Agents Chemother, v. 43, n. 2, p. 415-7, 1999.

69. MÜLLER-PREMRU, M.; LEJKO-ZUPANC, T. Epidemiological typing of imipenem-resistant Pseudomonas aeruginosa. International Journal of Antimicrobial Agents, v. 20, n. 5, p. 380-3, 2002.

70. MURPHY, T. A. et al. Crystal structure of Pseudomonas aeruginosa SPM-1 provides insights into variable zinc affinity of metallo-b-lactamases. Journal of Molecular Biology, v. 357, n. 3, p. 890-903, 2006.

71. NEVES, P. R. Alterações da permeabilidade e expressão de bombas de efluxo em isolados clínicos de Pseudomonas aeruginosa resistente ao imipenem. 2010. Tese (Doutoramento) - Faculdade de Ciências Farmacêuticas, Universidade de São Paulo.

72. NEVES, P. R. et al. Multirresistência mediada por metalo-beta-lactamases, porinas, bombas de efluxo e metilases, em isolados clínicos de Pseudomonas aeruginosa. In: XI CONGRESSO Brasileiro de Controle de Infecção e Epidemiologia Hospitalar, Rio de Janeiro. Braz J Infec Dis, v. 12, p. 30, 2008.

73. NICASIO, A. M. et al. The current state of multidrugresistant Gram-negative bacilli in North America. Pharmacotherapy, v. 28, n. 2, p. 235-49, 2008.

74. NIKAIDO, H. Porins and specific diffusion channels in bacterial outer membranes. J Biol Chem, v. 269, n. 6, p. 3905-8, 1994.

75. NIKAIDO, H. et al. Identification and characterization of porins in Pseudomonas aeruginosa. J Biol Chem, v. 266, n. 2, p. 770-9, 1991.

76. OCHIS, M. et al. Negative regulation of the Pseudomonas aeruginosa outer membrane porin OprD selective for imipenem and basic amino acids. Antimicrob Agents Chemother, v. 43, n. 5, p. 1085-90, 1999.
77. OH, E. J. et al. Prevalence of metallo- $\beta$-lactamase among Pseudomonas aeruginosa and Acinetobacter baumannii in a Korean university hospital and comparison of screening methods for detecting metallo- $\beta$-lactamase. J Microbiol Methods, v. 54, n. 3, p. 411-8, 2003.

78. OLIVEIRA, M. S. et al. Polymyxin B and colistimethate are comparable as to efficacy and renal toxicity. Diagn Microbiol Infect Dis, v. 65, n. 4, p. 431-4. 2009.

79. PAGĖS, J. M. et al. The porin and the permeating antibiotic: a selective diffusion barrier in Gram-negative bacteria. Nat Rev Microbiol, v. 6, n. 12, p. 893-903, 2008.

80. PEARSON, J. P. et al. Active efflux and diffusion are involved in transport of Pseudomonas aeruginosa cell-to-cell signals. J Bacteriol, v. 181, n. 4, p. 1203-10, 1999.

81. PELLEGRINO, F. L. et al. Occurrence of a multidrugresistant Pseudomonas aeruginosa clone in different hospitals in Rio de Janeiro, Brazil. J Clin Microbiol, v. 40, n. 7, p. 2420-4, 2002.

82. PICÃO, R. C. et al. Metallo-beta-lactamase detection: comparative evaluation of double-disk synergy versus combined disk tests for IMP-, GIM-, SIM-, SPM-, or VIM-producing isolates. J Clin Microbiol, v. 46, n. 6, p. 2028-37, 2008.

83. PIDDOCK, L. J. Clinically relevant chromosomally encoded multidrug resistance efflux pumps in bacteria. Clin Microbiol Rev, v. 19, n. 2, p. 382-402, 2006.

84. POIREL, L. etal. Carbapenem-hydrolyzing metallo- $\beta$-lactamase from a nosocomial isolate of Pseudomonas aeruginosa in France. Emerg Infect Dis, v. 6, n. 1, p. 84-5, 2000.

85. POIREL, L. et al. Characterization of bla ${ }_{D I M-1}$, a novel integronlocated metallo- $\beta$-lactamase gene from a Pseudomonas stutzeri clinical isolate in the Netherlands. $19^{\text {th }}$ European Congress of Clinical Microbiology and Infectious Diseases (ECCMID). Helsinki, Finland, 2009. v. O309.

86. POOLE, K. Efflux-mediated antimicrobial resistance. J Antimicrob Chemother, v. 56, n. 1, p. 20-51, 2005.

87. POOLE, K. etal. Expression of the multidrug resistance operon mexA-mexB-oprM in Pseudomonas aeruginosa: mexR encodes a regulator of operon expression. Antimicrob Agents Chemother, v. 40, n. 9, p. 2021-8, 1996b.

88. POOLE, K. et al. Multiple antibiotic resistance in Pseudomonas aeruginosa: evidence for involvement of an efflux operon. J Bacteriol, v. 175, n. 22, p. 7363-72, 1993.

89. POOLE, K. et al. Overexpression of the mexC-mexD-oprJ efflux operon in nfxB-type multidrug-resistant strains of Pseudomonas aeruginosa. Mol Microbiol, v. 21, n. 4, p. 713-24, 1996a.

90. RÅDBERG, G. et al. Development of quinoloneimipenem cross resistance in Pseudomonas aeruginosa during exposure to ciprofloxacin. Antimicrob Agents Chemother, v. 34, n. 11, p. 2142-7, 1990.

91. RUPP, M. E.; FEY, P. D. Extended spectrum beta-lactamase (ESBL)-producing Enterobacteriaceae: considerations for diagnosis, prevention and drug treatment. Drugs, v. 63, n. 4, p. 353-65, 2003.

92. SADER, H. S. et al. Epidemiologic typing of multiply drug-resistant Pseudomonas aeruginosa isolated from an outbreak in an intensive care unit. Diagn Microbiol Infect Dis, v. 17, n. 1, p. 13-8, 1993.

93. SADER, H. S. et al. IMPs, VIMs and SPMs: the diversity of metallo-beta-lactamases produced by carbapenem- 
resistant Pseudomonas aeruginosa in a Brazilian hospital. Clin Microbiol Infect, v. 11, n. 1, p. 73-6, 2005.

94. SADER, H. S. et al. Pathogen frequency and resistance patterns in Brazilian hospitals: summary of results from three years of the SENTRY Antimicrobial Surveillance Program. Braz J Infect Dis, v. 5, n. 4, p. 200-14, 2001.

95. SAFDAR, N. et al. Does combination antimicrobial therapy reduce mortality in Gram-negative bacteraemia? A metaanalysis. Lancet Infect Dis, v. 4, n. 8, p. 519-27, 2004.

96. SAIER JR, M. H. et al. Evolutionary origins of multidrug and drug-specific efflux pumps in bacteria. FASEB J, v. 12, n. 3, p. 265-74, 1998.

97. SEKIGUCHI, J. I. et al. KHM-1, a novel plasmid-mediated metallo-\{beta\}-lactamase from a Citrobacter freundi clinical isolate. Antimicrob Agents Chemother, v. 52, n. 11, p. $4194-7,2008$.

98. SOBEL, M. L. et al. Contribution of the MexXY multidrug transporter to aminoglycoside resistance in Pseudomonas aeruginosa clinical isolates. Antimicrob Agents Chemother, v. 47, n. 10, p. 3202-7, 2003.

99. SRIKUMAR, R. et al. Influence of mutations in mexR repressor gene on expression of the Mex A-MexB-OprM multidrug efflux system of Pseudomonas aeruginosa. J Bacteriol, v. 182, n. 5, p. 1410-4, 2000.

100. TAMBER, S. et al. Role of the novel OprD family of porins in nutrient uptake in Pseudomonas aeruginosa. J Bacteriol, v. 188, n. 1, p. 45-54, 2006.

101. TAMBER, S.; HANCOCK, R. E. W. Involvement of two related porins, OprD and OpdP, in the uptake of arginine by Pseudomonas aeruginosa. FEMS Microbiol Lett, v. 260, n. 1, p. 23-9, 2006.

102. TOLEMAN, M. A. et al. Molecular characterization of SPM-1, a novel metallo-beta-lactamase isolated in Latin America: report from the SENTRY antimicrobial surveillance programme. J Antimicrob Chemother, v. 50, n. 5, p. 673-9, 2002.

103. UNCHALEE TATTAWASART, J. Y. et al. Outer membrane changes in Pseudomonas stutzeri resistant to chlorhexidine diacetate and cetylpyridinium chloride. International Journal of Antimicrobial Agents, v. 16, n. 3, p. 233-8, 2000.

104. VIEIRA, V. V. et al. Metalo-B-lactamase produced by carbapenem-resistant Pseudomonas aeruginosa in Brazil. Clin Microbiol Infect, v. 11, n. 11, p. 937, 2005.

105. VILA, J.; MARCO, F. Interpretative reading of the non-fermenting Gram negative bacilli antibiogram. Enfermage Infectious Microbiology Clinical, v. 20, n. 6, p. 304-10, 2002.

106. WACHINO, J. et al. Mode of transposition and expression of $16 \mathrm{~S}$ rRNA methyltransferase gene rmtC accompanied by ISEcp1. Antimicrob Agents Chemother, v. 50, n. 9, p. 3212-5, 2006.

107. WALSH, T. R. et al. Evaluation of a new Etest for detecting metallo- $\beta$-lactamases in routine clinical testing. J Clin Microbiol, v. 40, n. 8, p. 2755-9, 2002.

108. WALSH, T. R. et al. Metallo-beta-lactamases: the quiet before the storm? Clin Microbiol Rev, v. 18, n. 2, p. 306-25, 2005.

109. WATANABE, M. et al. Transferable imipenem resistance in Pseudomonas aeruginosa. Antimicrob Agents Chemother, v. 35, n. 1, p. 147-51, 1991.
110. WOLTER, D. J. et al. Insertional inactivation of oprD in clinical isolates of Pseudomonas aeruginosa leading to carbapenem resistance. Fems Microbiology, v. 236, n. 1, p. 137-43, 2004.

111. XAVIER, D. E. Avaliação da expressão de sistemas de efluxo para a resistência antimicrobiana entre amostras clínicas de Pseudomonas aeruginosa. 2008. Dissertação (Mestrado) - Escola Paulista de Medicina, Universidade Federal de São Paulo.

112. YAMANE, K. et al. 16S rRNA methylase-producting, Gram-negative pathogens, Japan. Emerging Infectious Diseases, v. 13, n. 4, p. 642-6, 2007.

113. YOKOYAMA, K. et al. Acquisition of $16 \mathrm{~S}$ rRNA methylase in Pseudomonas aeruginosa. Lancet, v. 362, n. 9399, p. 1888-93, 2003.

114. YONEDA, K. et al. Measurement of Pseudomonas aeruginosa multidrug efflux pumps by quantitative real-time polymerase chain reaction. FEMS Microbiol Lett, v. 243, n. 1, p. 125-31, 2005.

115. YONEYAMA, H. et al. Nucleotide sequence of the protein D2 gene of Pseudomonas aeruginosa. Antimicrob Agents Chemother, v. 36, n. 8, p. 1791-3, 1992.

116. YONEYAMA, H. et al. Role of porins in the antibiotic susceptibility of Pseudomonas aeruginosa: construction of mutants with deletions in the multiple porin genes. Biochemical and Biophysical Research Communications, v. 213, n. 1, p. 88-95, 1995.

117. YONEYAMA, H.; NAKAE, T. Mechanism of efficient elimination of protein D2 in outer membrane of imipenemresistant Pseudomonas aeruginosa. Antimicrob Agents Chemother, v. 37, n. 11, p. 2385-90, 1993.

118. YONG, D. et al. A novel sub-group metallo-beta-lactamase $(M B L), A / M-1$ emerges in Pseudomonas aeruginosa (PSA) from Australia. 47 $7^{\text {th }}$ Interscience Conference in Antimicrobial Agents and Chemotherapy (ICAAC). Chicago, IL, USA, 2007. n. C1-539.

119. YONG, D. et al. Characterization of a new metallo-\{beta\}lactamase gene, blaNDM-1, and a novel erythromycin esterase gene carried on a unique genetic structure in Klebsiella pneumoniae sequence type 14 from India. Antimicrob Agents Chemother, v. 53, n. 12, p. 5046-54, 2009.

120. YOSHIHARA, E. et al. Protein D2 porin of the Pseudomonas aeruginosa outer membrane bears the protease activity. FEBS Letters, v. 394, n. 2, p. 179-82, 1996.

121. ZAVASCKI, A. P. et al. High rate of antimicrobial resistance in Pseudomonas aeruginosa at a tertiary-care teaching hospital in southern Brazil. Infect Control Hosp Epidemiol, v. 25, n. 10, p. 805-7, 2004.

122. ZAVASCKI, A. P. et al. The influence of metallo-Blactamase production on mortality in nosocomial Pseudomonas aeruginosa infections. J Antimicrob Chemother, v. 58, n. 2, p. 387-92, 2006.

\begin{tabular}{l|l} 
& Endereço para correspondência \\
\hline Patrícia R. Neves \\
Departamento de Microbiologia - ICBII/USP \\
Laboratório de Resistência Bacteriana e Alternativas Terapêuticas \\
Av. Prof. Lineu Prestes, 1.374/sala 240 - Butantã \\
CEP: 05508-000 - São Paulo-SP
\end{tabular}

